\title{
Beyond folk psychology
}

\author{
Steven Pinker
}

The Elm and the Expert. By Jerry A. Fodor. MIT Press: 1994. Pp. 129. \$19.95, $£ 15.95$.

BBC TELEVISION recently broadcast a debate on whether "science can explain human behaviour". Arguing against the resolution was a philosopher who asked how we might explain why John went to jail. Say it was for inciting racial hatred. The intention, the hatred and even the prison cannot be described in the language of physics, and the event can never be explained in terms of the movement of particles. So behaviour does not sit atop a hierarchy of levels resting on physics. Explanations of behaviour are narratives couched in the intentions of actors, a plane completely separate from natural science.

She had a point: people act according to their beliefs and desires. But she did not realize that narrative explanation, or folk-psychology, has for decades been firmly connected to science by philosophers of mind, most notably Jerry Fodor. The Elm and the Expert is his latest installment.

Fodor defends three hypotheses. First, behaviour is indeed explained by beliefs and desires, which are propositions that a person thinks are true or wants to be true. Second, these propositions are implemented in the brain as data structures organized like sentences, not in English but in a language of thought, 'mentalese'. Thinking is computation; mentalese symbols are rearranged by neural algorithms sensitive to the symbols' identities and configuration (their 'syntax').

What, then, gives mentalese its content (its 'semantics') - how do mental symbols stand for a proposition about the world that is true or false? A possible answer is Fodor's third hypothesis: mental content is information in something like the mathematical sense. The mental symbol 'dog' carries information about dogs because it correlates with the presence of dogs, thanks to the mechanisms of perception: under normal viewing conditions, being in the presence of a dog causes 'dog' to light up in the brain. The mechanisms that manipulate mentalese implement valid rules of inference, so that true inputs result in true outputs. So when all goes well, the world causes us to have mentalese sentences whose contents are true, and the brain's algorithms grind out new sentences whose contents are also true. Physics results in intelligence, the mind-body problem is solved and science can explain human behaviour.

But what happens when all does not go well? Fodor grapples mainly with two

longstanding objections to this happy picture, cases where the same mental symbol has different world contents, or vice versa. First, imagine a planet that resembles Earth in every way except that what looks and acts like water is chemically not $\mathrm{H}_{2} \mathrm{O}$ but XYZ. John and his Twin-Earth doppelgänger have identical mentalese, so are psychologically indistinguishable. But when thirsty they think about different things: $\mathrm{H}_{2} \mathrm{O}$ and XYZ. Either contents are not correlations with the world after all or contents make no difference to computations inside a head and so are irrelevant to psychology. Second, say John doesn't realize that 'the morning star' and 'the evening

\section{"Why the self-defeating hostility to natural selection? . . . That Fodor should swim away from this lifesaver is testimony to the contemporary allergy to Darwin in the humanities and cognitive sciences"}

star' are the same thing, Venus. He would have different symbols in mentalese, but their content is the same. More poignantly, recall Oedipus. $\mathrm{He}$, too, had distinct mentalese symbols with the same contents: 'Jocasta' and 'Mom'.

Fodor's solution may surprise philosophers: he dismisses their standard instrument, the thought experiment, and resists the demand for an exceptionless definition of content. Sure, he says, it's possible for thoughts and world states not to correlate, but it doesn't happen very often; only in special circumstances, as an unsystematic exception to general laws. After all, the facts of chemistry surely rule out there actually being an XYZ that is indistinguishable from our water. And, for humans to be as clever as they are, then surely, whenever the consequences matter, they gather enough information not to make morning star/evening star confusions. Oedipus, shmoedipus - he was so unlucky, Sophocles wrote a play about the coincidences that did him in.

Fodor's solution should not surprise scientists. He is saying that mental content is not an a priori logical concept but part of an empirical claim about the human brain. Indeed, his solution fits easily into a Darwinian psychology. We have thoughts about the world because that's what natural selection had to give us to get our onboard computers to make inferences that are useful in that world. Thoughts and world can sometimes fall out of sync because selection adapts organisms only to their typical worlds, not to all worlds. Colour vision, taste and sexuality don't work as designed in a world with sodium lamps, saccharin and contraception, and cognition doesn't work as designed in the worlds thought up by philosophers.

But here comes a shocker. Fodor is contemptuous of the suggestion that any "song and dance about Darwin" is relevant. He writes: "evolution maybe explains why there are more things around that work than there are things that don't. But it doesn't explain how things work, and it is decisively a 'how' question that we're faced with. So please, spare me; no Darwin."

The sarcasm is unseemly. Of course natural selection doesn't explain how things work in the sense of proximate causation inside a given organism. But Fodor doesn't explain how things work either. To do that, he would have to be doing actual psychology: specifying the computations that human minds perform. In fact, he is reasoning not from 'how' but from 'why' - in his words, "why God bothered to give us [minds]", "what perception and cognition are for", "[w]hy we are so clever", "why having a mind is a good idea". That is, he invokes the design that an organ, in this case the mind, has to have to function adaptively in its standard environment. This is precisely the kind of question that requires a song and dance about Darwin - especially in a book whose main point is to dismiss counter-examples because they involve species-atypical circumstances.

Why the self-defeating hostility to natural selection? I think Fodor misunderstands its explanatory power. It is simply wrong to say that selection just explains contemporary demographics, "why there are more things around that work than there are things that don't". When the demographics are iterated over millions of generations of mutation and replication, natural selection explains how there can be anything around that works to begin with. That Fodor should swim away from this lifesaver is testimony to the contemporary allergy to Darwin in the humanities and cognitive sciences.

But when reading Fodor, outlandish twists are all part of the fun, for they always alternate with ingenious twists and are presented with an inimitable, irreverent wit. Although you may need a philosopher friend to explain the argot and allusions, The Elm and the Expert is a stimulating and intermittently convincing story about one of our deepest questions, the scientific basis of mind and meaning.

Steven Pinker is in the Center for Cognitive Neuroscience, Massachusetts Institute of Technology, Cambridge, Massachusetts 02139, USA. 